\title{
Publisher Correction: Ferroelastic-switching-driven large shear strain and piezoelectricity in a hybrid ferroelectric
}

Yuzhong Hu(D), Lu You (D), Bin Xu DD, Tao Li, Samuel Alexander Morris, Yongxin Li, Yehui Zhang, Xin Wang, Pooi See Lee (iD, Hong Jin Fan (D) and Junling Wang (D)

Correction to: Nature Materials https://doi.org/10.1038/s41563-020-00875-3, published online 11 January 2021.

In the version of this Letter originally published, in the key in Fig. 3e the red data were mistakenly labelled 'PMM-0.3PT(100)'; the label should have been 'PMN-0.3PT(100)'. This has now been corrected.

Published online: 18 January 2021

https://doi.org/10.1038/s41563-021-00929-0

() The Author(s), under exclusive licence to Springer Nature Limited 2021

\section{Publisher Correction: Conductivity control via minimally invasive anti-Frenkel defects in} a functional oxide

Donald M. Evans (D), Theodor S. Holstad, Aleksander B. Mosberg (D), Didrik R. Småbråten (D), Per Erik Vullum, Anup L. Dadlani (D), Konstantin Shapovalov, Zewu Yan, Edith Bourret, David Gao, Jaakko Akola, Jan Torgersen (D, Antonius T. J. van Helvoort, Sverre M. Selbach (D) and Dennis Meier (D)

Correction to: Nature Materials https://doi.org/10.1038/s41563-020-0765-x, published online 17 August 2020.

In the version of this Article originally published, the author Jan Torgersen was mistakenly affiliated with Institut de Ciència de Materials de Barcelona (ICMAB-CSIC), Campus UAB, Bellaterra, Spain; the affiliation has now been corrected to Department of Mechanical and Industrial Engineering, Norwegian University of Science and Technology (NTNU), Trondheim, Norway.

Published online: 18 February 2021

https://doi.org/10.1038/s41563-021-00956-X

๑) The Author(s), under exclusive licence to Springer Nature Limited 2021 